Characterization of the ion beam formed in a low magnetic field helicon mode

This article has been downloaded from IOPscience. Please scroll down to see the full text article.

2011 J. Phys. D: Appl. Phys. 44145204

(http://iopscience.iop.org/0022-3727/44/14/145204)

View the table of contents for this issue, or go to the journal homepage for more

Download details:

IP Address: 150.203.181.215

The article was downloaded on 23/03/2011 at 01:52

Please note that terms and conditions apply. 


\title{
Characterization of the ion beam formed in a low magnetic field helicon mode
}

\author{
T Lafleur, C Charles and R W Boswell \\ Space Plasma, Power and Propulsion Group, Research School of Physics and Engineering,The \\ Australian National University, Canberra ACT 0200, Australia \\ E-mail: trevor.lafleur@anu.edu.au
}

Received 22 August 2010, in final form 6 January 2011

Published 22 March 2011

Online at stacks.iop.org/JPhysD/44/145204

\begin{abstract}
An experimental characterization of the ion beam formed during a low magnetic field $(<3 \mathrm{mT})$ helicon mode has been performed using a number of retarding field energy analysers, for pressures between $0.03 \mathrm{~Pa}<p_{0}<0.4 \mathrm{~Pa}$ in argon. The low-field helicon mode is formed over a narrow magnetic field range $\left(1 \mathrm{mT}<B_{0}<3 \mathrm{mT}\right)$ using a single solenoid giving a strong diverging field, and is characterized by a distinct density peak. For pressures below about $0.3 \mathrm{~Pa}$, an ion beam is observed in the downstream region of the plasma reactor, and its presence is directly correlated with that of the low-field mode, suggesting that the ion beam formation is induced by the appearance of the helicon mode. For the same pressure and rf power input, as the magnetic field is further increased, a second ion beam regime is observed for magnetic fields above $5 \mathrm{mT}$. In this second regime the plasma density is less than half that for the low-field mode, and the system seems most likely to be inductively coupled. A simple analytical model is developed to describe the general features and behaviour of the observed ion energy distribution functions as a function of the plasma potential profile and neutral gas pressure.
\end{abstract}

(Some figures in this article are in colour only in the electronic version)

\section{Introduction}

The recent discovery of energetic ions formed in expanding and double-layer plasmas [1,2] has sparked a large amount of research, experimentally [3-9], theoretically [10-12] and numerically $[13,14]$ to understand the ion acceleration processes, and related physics in these low pressure plasmas. While some of these studies have focused on astrophysical phenomena [15] such as the aurora [16], the majority have typically been performed with propulsion applications in mind. Although these energetic ions (which are directional, and thus constitute an ion beam) can be formed in plasmas expanded purely geometrically $[17,18]$, a diverging magnetic field is usually needed, with field strengths of the order of $10 \mathrm{mT}$ $[3,4,6-9]$. This expansion is necessary in order to produce a large density gradient between the upstream and downstream plasma regions [19], which results in a drop in the plasma potential, wherein which the ions are accelerated. Regardless of the means of producing the expansion though, these ion beams are usually only observed at low pressures $(<0.3 \mathrm{~Pa}$ for argon) $[2,6,18]$.
Double-layer formation and plasma expansion have been observed in systems with a number of different heating mechanisms including inductive [3,4], helicon [1,20] and electron cyclotron resonance (ECR) sources [21]. The formation of an ion beam is typically insensitive to the type of heating mechanism, so long as a sufficient density gradient is present to produce the change in plasma potential needed to accelerate the ions. Of the heating mechanisms used however, helicon discharges are very attractive, since they are characterized by large power transfer efficiencies between the rf antenna and plasma, and in general are able to produce higher densities when compared with other types of heating mechanisms, such as inductive [22-24]. While higher densities are not always advantageous for propulsion applications, since they can lead to increased erosion rates of plasma source components [25,26], they are useful from the stand point that a higher density in a given reactor can produce a larger thrust density, as well as the fact that larger densities for a given mass flow rate of propellant corresponds to larger propellant utilization fractions [26] (that 
is, more of the propellant is usefully used before leaving the thruster).

In helicon theory, for an rf antenna with a given length (which consequently most strongly excites a particular helicon wavelength), the plasma density is proportional to the applied magnetic field [22]. At low magnetic fields ( $<5 \mathrm{mT})$ though, a density peak is usually seen occurring over a narrow range of field values [20,27-30]. This is typically referred to as a low-field helicon mode. Since the magnetic field is lower, a smaller density is needed to initiate and sustain the mode, which in principle allows these modes to operate at lower power inputs. Low-field helicons are potentially attractive for propulsion [31] or processing applications [32] since the lower required magnetic field means lower system/hardware mass and power consumption. Studies of ion acceleration and plasma expansion in these low-field modes have at present been very limited, since the majority of studies have been performed in uniform magnetic fields. In these uniform field cases, plasma density profiles are typically more uniform, and no ion beam formation has been observed.

We have recently reported the existence of an ion beam together with a low-field helicon mode in the presence of a diverging magnetic field [20]. The ion beam is produced when the plasma undergoes a direct capacitive to wave mode transition, during which the plasma changes from a roughly uniform state into an expanding plasma containing the ion beam. However, the connection between the presence of the ion beam and the low-field mode was not completely investigated, and no beam characterization was performed. Since almost no other work exists in the literature focusing on these factors in low-field helicon discharges, the present investigation aims to address this by performing a characterization of the ion beam both spatially, and parametrically. We also develop a simple analytical model to predict the general features of the energy distribution of these ion beams, and find that the gradient of the plasma potential profile and neutral gas pressure are important factors in determining the final shape of the energy distribution.

\section{Apparatus}

The present investigation makes use of the Piglet helicon reactor, a schematic of which is shown in figure 1 . The reactor and $\mathrm{rf}$ circuit have been described in detail before [20,33], and in short consists of a Pyrex source tube connected to a larger aluminium diffusion chamber. The source tube is $20 \mathrm{~cm}$ long and has a diameter of $13.6 \mathrm{~cm}$, while the diffusion chamber has a length of $28.8 \mathrm{~cm}$ and a diameter of $32 \mathrm{~cm}$. One end of the source tube is terminated with a grounded metal grid (which is then connected to a rotary/turbomolecular vacuum pump system), while the other end opens into the diffusion chamber. Surrounding the source tube is a $10 \mathrm{~cm}$ long double-saddle field antenna which is used to produce the plasma. Surrounding both the antenna and source tube is a grounded metal support structure that houses two solenoids, each with approximately 500 turns. The solenoid closest to the pump is termed the source coil, while the other coil is termed the exhaust coil (see figure 1). The antenna is air cooled, and is connected

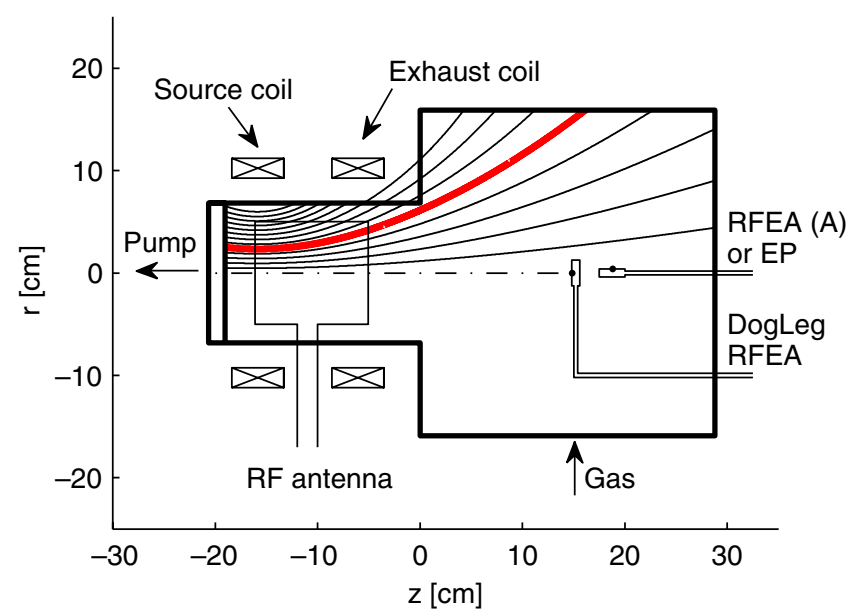

Figure 1. Schematic of the Piglet helicon reactor, showing the source region $(z<0 \mathrm{~cm})$, diffusion chamber $(z>0 \mathrm{~cm})$ and magnetic field coils. The magnetic field lines for the source coil (exhaust coil off) are shown above the central dashed line. The bold red line shows the outermost magnetic field line that just exits without intersecting the source tube wall.

to a matching network and $\mathrm{rf}$ power generator operated at 13.56 MHz. Argon gas enters the reactor via a port in the side of the diffusion chamber, and the pressure is measured with a baratron gauge. Diagnostics probes are inserted into ports in the backplate or side wall of the diffusion chamber. These probes can translate axially (or radially if inserted into a side port) without breaking vacuum integrity. Unless otherwise specified, all experiments in this study are performed at a total rf power input of $250 \mathrm{~W}$.

\section{Diagnostics}

Two types of electrostatic probes are used in this study, retarding field energy analysers (RFEAs) and an emissive probe (for a representative example of the insertion location of these probes, see figure 1). The RFEA has been described in detail previously $[8,34]$, and is used to measure the local plasma potential, as well as to determine the ion energy distribution function (IEDF) [3], and hence detect the presence of an ion beam. The RFEA consist of a grounded metal head with an orifice to let the plasma in. Located inside the head assembly are a number of biased grids that serve to repel electrons and discriminate the energy of any ions which enter. This discriminator voltage, $V_{\mathrm{d}}$, is swept from 0 to $80 \mathrm{~V}$. The RFEA is a directional probe, and it only measures ions which enter through the front orifice. RFEA (A) in figure 1 is situated on Piglet's central axis, with its orifice facing the side walls of the reactor. This RFEA cannot see an ion beam (since its orifice is facing away from the source region), and is used only to determine the local value of the plasma potential [3]. The 'dogleg' RFEA (with a $90^{\circ}$ bend in the support shaft) has its orifice facing towards the upstream source region, and is used to detect the presence of an ion beam, and take additional plasma potential measurements [3]. The RFEA measures an ion current as a function of $V_{\mathrm{d}}$. This current is then smoothed with a digital filter, and the IEDF is then obtained by taking the 


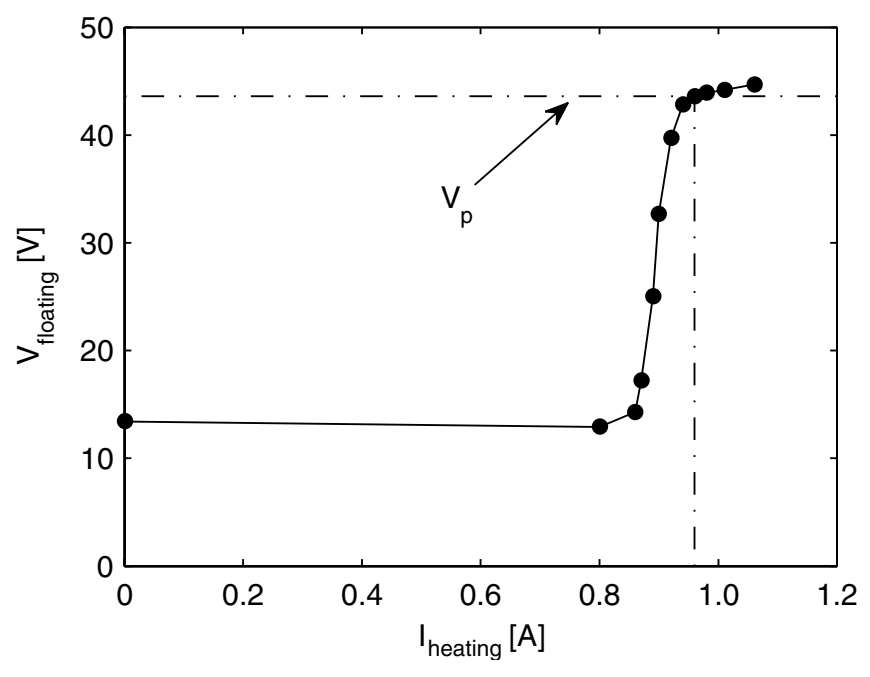

Figure 2. Floating potential, $V_{\text {floating }}$, of the emissive probe as a function of the applied heating current, $I_{\text {heating }}$. The horizontal dashed line indicates the plasma potential, which occurs at the knee of the curve, while the vertical dashed line is the heating current at this point.

negative derivative with respect to $V_{\mathrm{d}}$ [17]. Since the RFEA head is grounded, it should be noted that any ions which enter the probe have fallen through the sheath potential in front of the probe. The RFEAs described here are of a similar design to those in [34] which were shown to have an energy resolution of around $0.2 \mathrm{~V}$. The grid voltages here are slightly different so that this value could be higher, but the uncertainty is still expected to be less than $1 \mathrm{~V}$ [3].

The emissive probe (EP) is used to take additional independent measurements of the local plasma potential. The probe has been described before [8], and consists of a small $0.125 \mathrm{~mm}$ diameter tungsten wire inserted into two holes in a small ceramic tube. Copper wires inside these holes provide the heating current, which is supplied from a dc power supply (isolated from ground with an isolation transformer). If the heating current to the probe is large enough, then electron emission from the filament surface is sufficient to neutralize the sheath around the probe tip. When this happens the probe tip floats to the plasma potential [35]. This has recently been showed to be a reliable method to measure the plasma potential in the present system [8]. To determine the plasma potential, the floating potential, $V_{\text {floating }}$, of the probe is measured as a function of the applied heating current, $I_{\text {heating. An example }}$ is shown in figure 2. Initially there is no change in $V_{\text {floating, }}$ but as electron emission is initiated there is a rapid increase. As the heating current is further increased, $V_{\text {floating }}$ begins to level off to an approximately constant value. The plasma potential, $V_{\mathrm{p}}$, is defined as the knee of the second part of this curve, as demonstrated in figure 2 . This definition results in an uncertainty in the plasma potential of approximately $\pm 1 \mathrm{~V}$. In addition, for the heating currents needed to cause sufficient electron emission $(\approx 1-1.5 \mathrm{~A})$ a voltage drop of about $2 \mathrm{~V}$ occurs across the probe filament. Thus there is an additional uncertainty of $\pm 1 \mathrm{~V}$, giving a total combined uncertainty of about $\pm 2 \mathrm{~V}$.

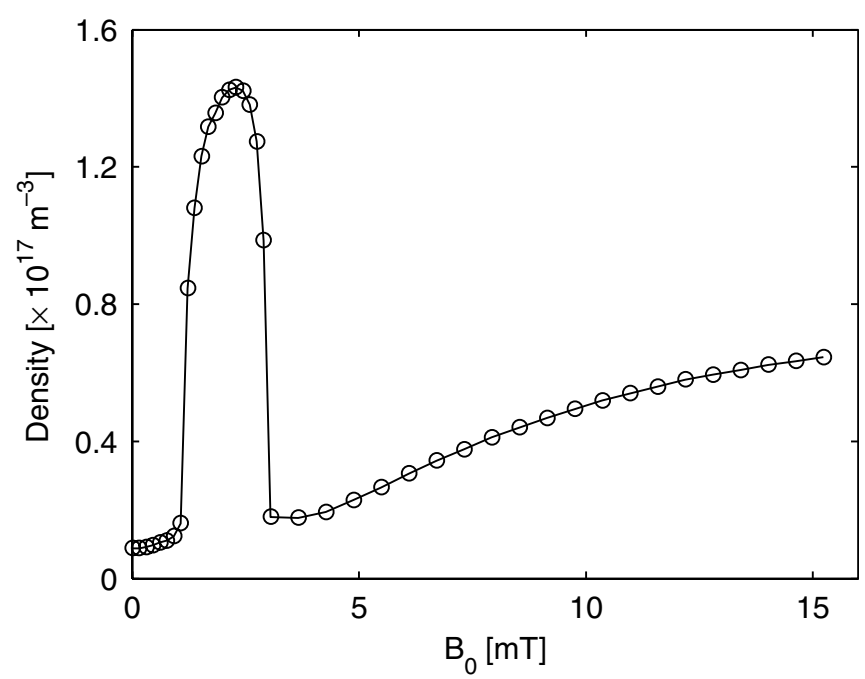

Figure 3. Plasma density within the source region $(z=-10 \mathrm{~cm})$ as a function of the maximum applied magnetic field (as the source coil is varied, with the exhaust coil off) for a pressure of $0.04 \mathrm{~Pa}$. The data in this figure have been taken previously by the authors, and is presented in [20].

\section{Results}

\subsection{Connection between low-field mode and ion beam}

With just the source coil operating (exhaust coil off; see figure 1) it has been shown that a low-field helicon mode exists within the present reactor [20]. Figure 3 shows the density within the source region at $z=-10 \mathrm{~cm}$ taken previously [20] with a Langmuir probe, as a function of the maximum magnetic field (since only the source coil is operating, a diverging magnetic field is present; see figure 1). For low magnetic fields, a large increase in the plasma density is seen over a very narrow range of magnetic field values $\left(1 \mathrm{mT}<B_{0}<3 \mathrm{mT}\right)$. This density peak in figure 3 is a low-field helicon mode. An ion beam has also previously been observed to occur together with this low-field mode at a maximum magnetic field of $2.1 \mathrm{mT}$ [20]; however, no direct connection was established between the two for other magnetic fields. It should be noted that although an ion beam was observed, it is almost certainly not due to a double layer. Measurement of the plasma potential [20] shows a drop of approximately $20 \mathrm{~V}$ over about $20 \mathrm{~cm}$, a far greater distance than the $1-2 \mathrm{~cm}$ observed in current-free double layers [2], this despite the larger densities encountered here (which would give a smaller Debye length). This suggests that the ion beam could be produced due to plasma expansion rather than by acceleration in a double layer.

To investigate the connection between the ion beam and the low-field mode, IEDFs are measured for magnetic fields below about $4 \mathrm{mT}$ with the 'dogleg' RFEA located at $z=10 \mathrm{~cm}$. These IEDFs are then normalized and combined to produce the contour plot shown in figure 4(a). In the absence of an ion beam, the IEDF is approximately Gaussian in shape, with a single peak at the local plasma potential. This is indeed seen for magnetic fields below $1 \mathrm{mT}$, with the plasma potential given by the dark band stretching from around $45 \mathrm{~V}$ to $40 \mathrm{~V}$ for $B_{0}<1 \mathrm{mT}$ (dark red regions online). As the 

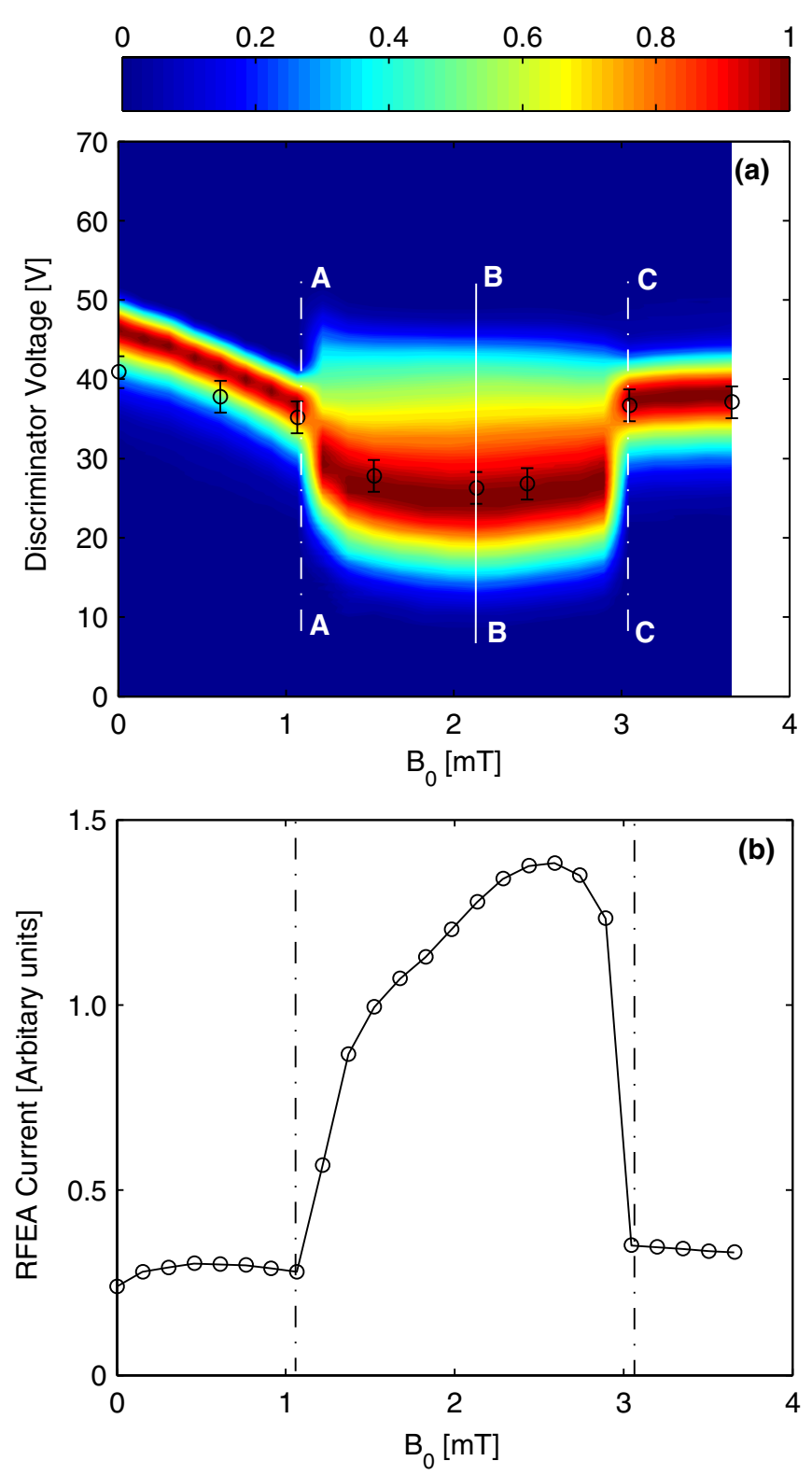

Figure 4. (a) IEDFs as a function of the maximum applied magnetic field for the cases in figure 3 with $B_{0}<3.7 \mathrm{mT}$, taken downstream (at $z=10 \mathrm{~cm}$ ) with the 'dogleg' RFEA for a pressure of $0.04 \mathrm{~Pa}$. The dark red regions are indications of the local plasma potential, while the open circles are the plasma potentials found with the emissive probe. The vertical dashed lines $A A$ and $C C$ correspond to the lower and upper limits of the low-field peak in figure 3 , while the vertical solid line $B B$ corresponds to the IEDF at the maximum of the low-field peak in figure 3. (b) Total ion current measured for the cases in $(a)$. The black vertical dashed lines correspond to the lower and upper limits of the low-field peak in figure 3 .

magnetic field is increased, however, a discontinuous change is observed at $1 \mathrm{mT}$, with the plasma potential decreasing from around $40 \mathrm{~V}$ to less than $30 \mathrm{~V}$, and the IEDFs becoming significantly asymmetric about the maximum value. This asymmetry gives an indication of the presence of a higher energy ion population. Further increases in the magnetic field show a second discontinuous change at around $3 \mathrm{mT}$, with the plasma potential rising to about $40 \mathrm{~V}$ and the IEDFs once again becoming symmetric around the plasma potential. We note that the discontinuous changes correlate well with the lower and upper magnetic field limits of the low-field density peak in figure 3, as indicated by the dotted lines $A A$ and $C C$ in figure 4(a). Furthermore, throughout the magnetic field range giving the low-field mode, a higher energy ion population is observed. Thus both occur simultaneously, appearing and disappearing together.

To check the plasma potential seen by the RFEA, independent measurements are made with the emissive probe described in section 3. The probe is inserted at the same location that the RFEA measurements were made, and the results (open circles) are overlaid on the contour plot in figure $4(a)$. With the exception of the zero field case, the plasma potentials from the EP match very well with those found from the 'dogleg' RFEA (dark red regions online), and in particular show that there is indeed two discontinuous jumps present. Figure $4(b)$ shows the total current collected by the RFEA (with a discriminator voltage of $0 \mathrm{~V}$ ) during the transition. For the low-field peak of figure 3, this total current is observed to increase. Thus during the low-field mode, in addition to an ion beam forming, the ion current also increases in the downstream region. Note that the increase in current (which is a measure of the density) during the low-field peak is not as large as that within the source region, since the density change in the downstream chamber is not as large [20].

If the asymmetric nature of the IEDFs discussed above were due to broadening effects associated with rf modulation of the sheath in front of the RFEA [36] and not due to ion acceleration in an expanding plasma, then by rotating the probe (so that the orifice no longer faces the source tube) these effects should still be present. To do this we insert RFEA (A) into a side port of the diffusion chamber, located at $z=15 \mathrm{~cm}$. Here we define $0^{\circ}$ when the orifice faces the source tube. Rotating the probe by $90^{\circ}$ then causes the orifice to face the side walls of the reactor. The IEDF results for different angles are shown in figure 5 for the cases denoted $B B$ and $C C$ in figure 4(a). These cases are for IEDFs during, and just after, the low-field peak. The IEDFs just before the peak are similar to those just after, and are omitted for clarity. At $0^{\circ}$, case $C C$ (dotted line) shows a single ion population (approximately Gaussian in shape), while $B B$ (solid line) shows a much broader distribution with a group of higher energy ions. As the probe is rotated these higher energy ions disappear, and for angles of $90^{\circ}, 180^{\circ}$ and $270^{\circ}$, only a single ion population is seen. This shows that the higher energy ion population is not due to $\mathrm{rf}$ broadening effects, but is directional, and hence is an ion beam. For all angles the IEDFs for case $C C$ remain the same. This test is important, since if the plasma density increases (as it does during the low-field mode) the Debye length decreases, and by considering ion motion through the sheath in front of the RFEA, the transit time would decrease [36]. If this transit time is less than or equal to an rf period, then any residual capacitive coupling would modulate the plasma potential, and this could affect the IEDFs, thus producing an artificial higher energy ion population. This, however, is clearly not the case here and thus the higher energy ion population is a directional beam.

Setting the magnetic field to $2.1 \mathrm{mT}$ (approximately the maximum of the density peak in figure 3 ), IEDFs are measured 

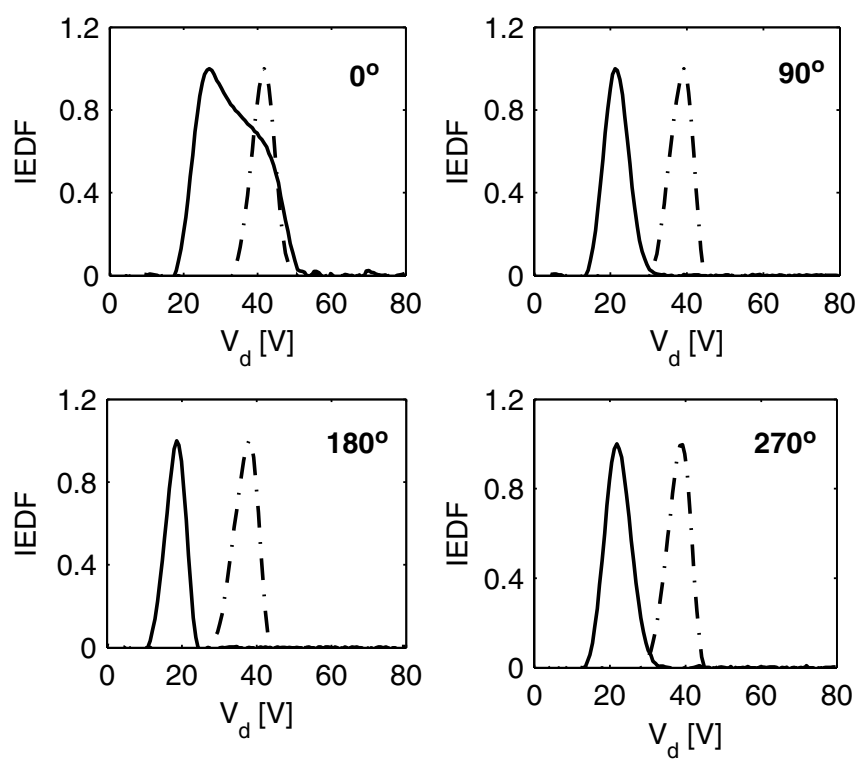

Figure 5. IEDFs for cases $B B$ (solid line) and $C C$ (dashed line) in figure $4(a)$ at a number of RFEA orifice rotation angles $\left(0^{\circ}\right.$ is defind when the RFEA orifice faces the upstream region and is normal to Piglet's central axis).

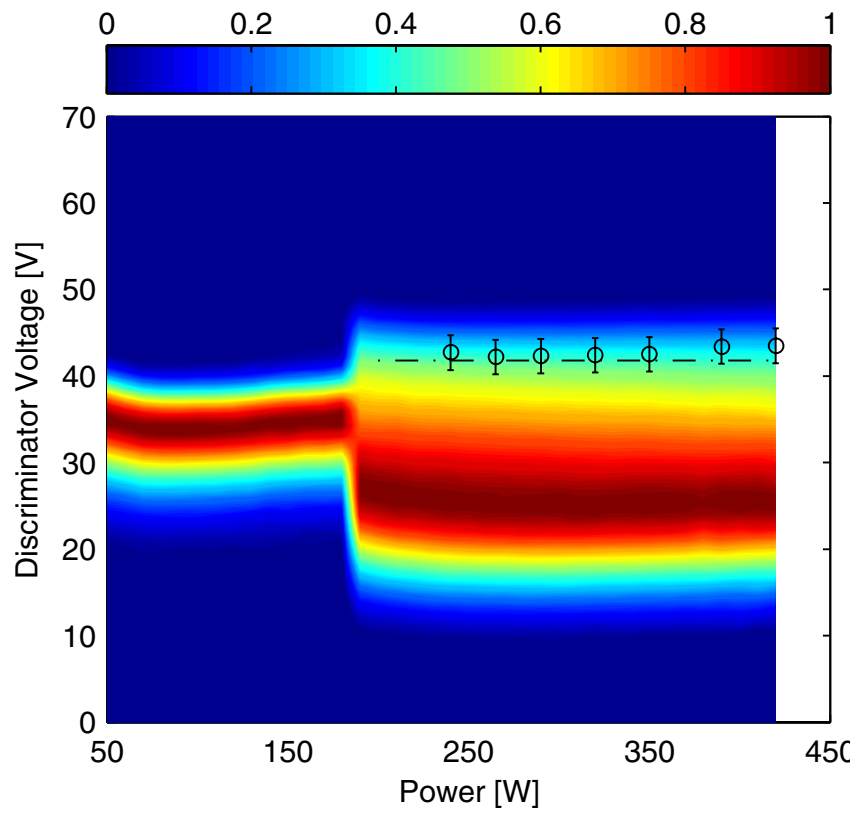

Figure 6. IEDFs as a function of the total $\mathrm{rf}$ power input, taken downstream (at $z=10 \mathrm{~cm}$ ) with the 'dogleg' RFEA for a pressure of $0.04 \mathrm{~Pa}$, and a maximum magnetic field (with just the source coil on) of $2.1 \mathrm{mT}$. The open circles are the plasma potentials found with the emissive probe, while the horizontal dashed line indicates the plasma potential upstream (at $z=-10 \mathrm{~cm}$ ) taken with RFEA (A).

with the 'dogleg' RFEA (located at $z=10 \mathrm{~cm}$ ) as the rf power input is increased. This is shown in figure 6. For powers less than about $200 \mathrm{~W}$, the IEDFs are approximately symmetric about the plasma potential, but at $200 \mathrm{~W}$ a discontinuous change occurs, and the plasma potential decreases. Here a higher energy ion population is again seen. At this transition magnetic field, the plasma changes from a dull purple to a bright pink colour in the source region, and the matching is

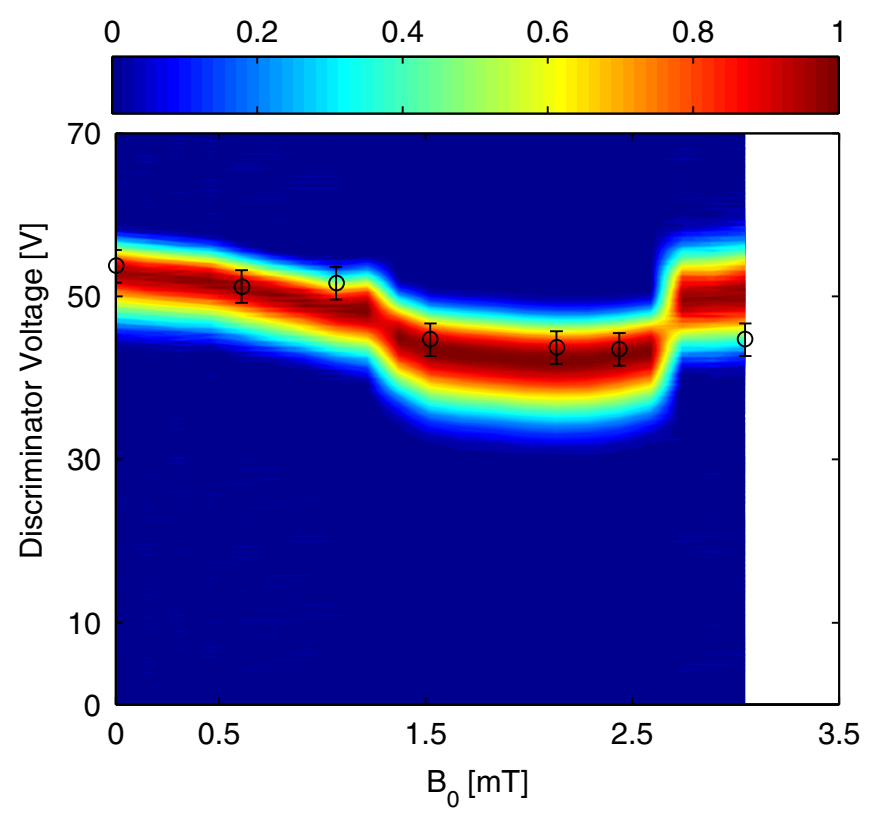

Figure 7. IEDFs as a function of the maximum applied magnetic field for the cases in figure 3 with $B_{0}<3 \mathrm{mT}$, taken upstream (at $z=-10 \mathrm{~cm}$ ) with RFEA (A) for a pressure of $0.04 \mathrm{~Pa}$. The dark red regions are indications of the local plasma potential, while the open circles are the plasma potentials found with the emissive probe.

observed to change significantly. This is the start of the lowfield mode. We thus see once again that the appearance of the low-field mode and the ion beam are connected. As the power is further increased, both the plasma potential and the IEDFs remain virtually identical. The only change observed is that the ion beam current (not shown) increases. This behaviour is perhaps not unexpected, since from a simple particle balance [24], at steady state the electron temperature is independent of the input power. Since the plasma potential is typically some function of the electron temperature (for noncapacitively coupled systems) [24], as the power increases we would not expect the plasma potential to change, only the density to increase.

The dotted line in figure 6 represents the upstream plasma potential at $z=-10 \mathrm{~cm}$ measured with RFEA (A). The upstream potential corresponds well to the energy of the higher energy ions in the IEDFs during the low-field mode. The open circles are measurements of the plasma potential made in the upstream region (also at $z=-10 \mathrm{~cm}$ ) with the EP. They correspond well to that from RFEA (A), and also show that the plasma potential does not increase as the power changes. However at higher powers $(>380 \mathrm{~W})$, the potentials begin to rise slightly, although still within the error bounds.

\subsection{Characterization of the plasma potential}

With RFEA (A) reinstalled along Piglet's central axis, plasma potential measurements are made in the source region as the magnetic field is increased. This is shown in figure 7. Since RFEA (A) now has its orifice facing the reactor walls, it cannot see an ion beam, and hence the IEDFs are symmetric around the local plasma potential (dark red regions online). Two discontinuous changes are again seen as the magnetic field 


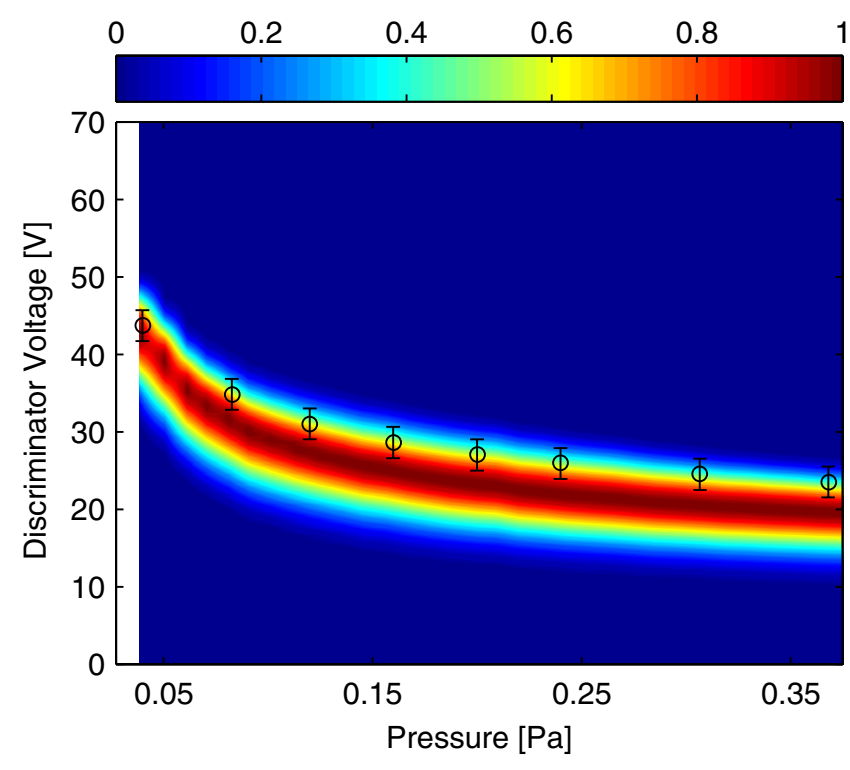

Figure 8. IEDFs as a function of neutral gas pressure, taken upstream (at $z=-10 \mathrm{~cm}$ ) with RFEA (A) for a maximum magnetic field (with just the source coil on) of $2.1 \mathrm{mT}$. The open circles are the plasma potentials found with the emissive probe.

is increased, one at about $1 \mathrm{mT}$, and a second at $2.5 \mathrm{mT}$. The plasma potential decreases from around $50 \mathrm{~V}$ down to $45 \mathrm{~V}$, before increasing again back to about $50 \mathrm{~V}$. Note that the second discontinuity occurs slightly early than that in figure 3 since the presence of the grounded probe in the source region perturbs the plasma to a larger extent. We note that the peak upstream potentials during the low-field mode are around $45 \mathrm{~V}$, which matches well with the average energy of the ion beam in figure $4(a)$. Also shown in figure 7 are additional EP measurements, which once again match well with the plasma potentials found with the RFEA.

Figure 8 shows the upstream IEDFs taken with RFEA (A) (located at $z=-10 \mathrm{~cm}$ ) as the pressure is changed. As the pressure increases the plasma potential (dark red regions online) decreases, going from around $45 \mathrm{~V}$ at $0.04 \mathrm{~Pa}$ to about $20 \mathrm{~V}$ at $0.36 \mathrm{~Pa}$. The open circles again represent the plasma potentials found with the EP. Although the trend displayed is similar to that from the RFEA, the potentials are a few volts higher for all pressures. It should be said though that the EP measurements were not taken simultaneously with the RFEA results (which themselves have an uncertainty). Nevertheless the results are still in reasonable agreement, and deviate by about $4 \mathrm{~V}$ at most.

\subsection{Characterization of the low-field ion beam}

Figure $9(a)$ shows IEDFs taken with the 'dogleg' RFEA in the downstream region (at $z=10 \mathrm{~cm}$ ) as a function of pressure (contour plots are not used so as to more clearly show the shape of the IEDFs). At low pressures $(<0.1 \mathrm{~Pa})$ there is a large higher energy ion population (with a broad range of energies) but as the pressure increases the IEDFs become more Gaussian in shape, and the higher energy population begins to disappear. For pressures greater than about $0.3 \mathrm{~Pa}$, almost no ion beam can be discerned. Of further interest is the fact that the IEDF
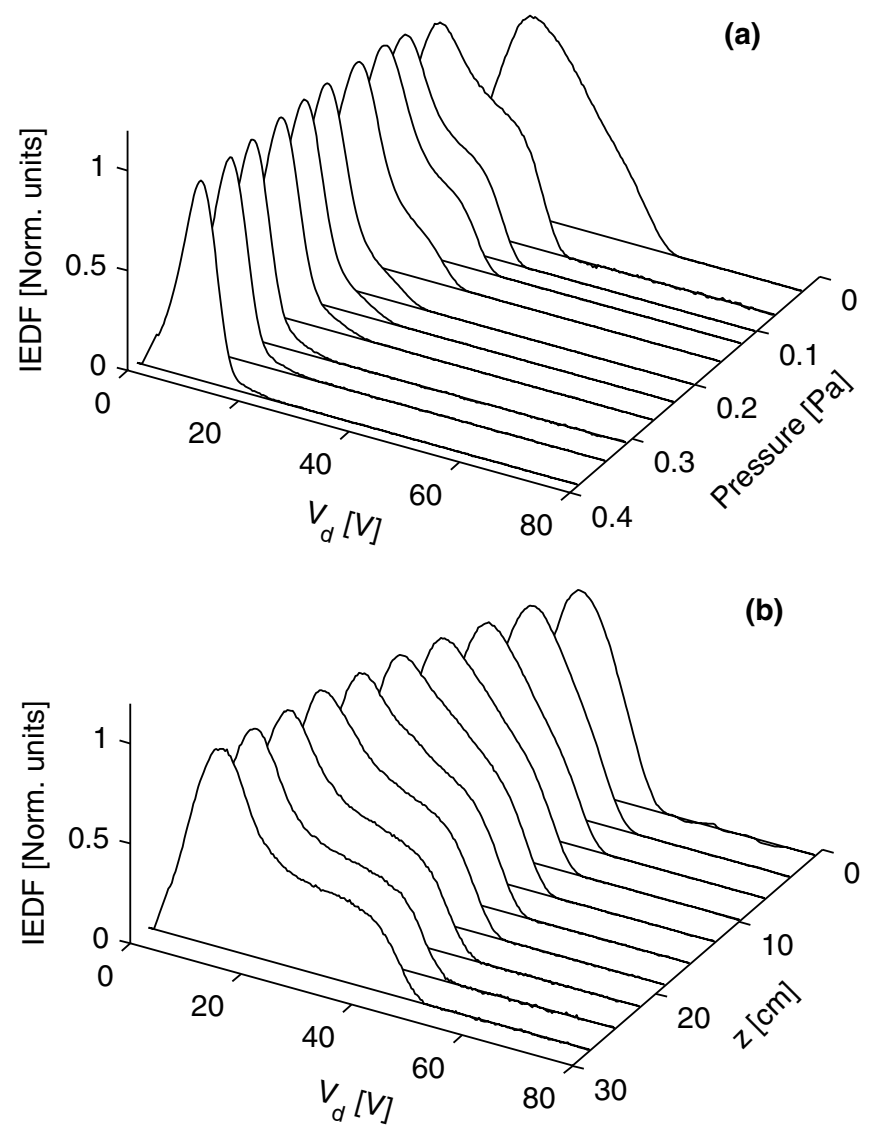

Figure 9. (a) IEDFs as a function of neutral gas pressure, taken downstream (at $z=10 \mathrm{~cm}$ ) with the 'dogleg' RFEA for a maximum magnetic field (with just the source coil on) of $2.1 \mathrm{mT}$. (b) IEDFs as a function of axial probe position, taken with the 'dogleg' RFEA, for a neutral gas pressure of $0.04 \mathrm{~Pa}$ and a maximum magnetic field (with just the source coil on) of $2.1 \mathrm{mT}$.

measurements in figure $9(a)$ were taken for magnetic fields strengths all producing a low-field mode [37]. Thus once again, this demonstrates a strong relationship between the presence of the low-field helicon mode and the ion beam.

The plasma potential (given approximately by the location of the peak of each of the IEDFs in figure $9(a)$ ) becomes smaller as the pressure increases (going from about 25 to $10 \mathrm{~V}$ ), as does the maximum energy of the ion beam population (which serves as an indication of the potential from which the ions were born in the upstream region). The 'decay' of the ion beam with pressure is a function of two main factors. Firstly, as the pressure increases the upstream plasma potential goes from around $45 \mathrm{~V}$ at $0.04 \mathrm{~Pa}$ (see figure 8), to around $20 \mathrm{~V}$ at $0.3 \mathrm{~Pa}$, while the plasma potential in the downstream region goes from around $25 \mathrm{~V}$ at $0.04 \mathrm{~Pa}$ to around $10 \mathrm{~V}$ at $0.3 \mathrm{~Pa}$. Thus the 'total' potential drop between the upstream and downstream regions decreases with pressure (a similar result has been seen in double-layer studies [12,38]). Secondly, as the pressure increases the mean free ion-neutral collision length becomes shorter. At the ion energies of interest (1-10 eV) charge exchange collisions are dominant (with a cross-section of around $0.6 \times 10^{-18} \mathrm{~m}^{2}$ [39]). In these collisions, an ion collides with a neutral gas atom and an electron jumps from the neutral to the ion, hence becoming an ion itself, and neutralizing the 
colliding ion. This then leaves a fast neutral and a slow ion, and thus affects the measured IEDFs (since the RFEA cannot detect the fast neutrals). The mean free path, $\lambda$, can be given by $\lambda=1 / n_{\mathrm{g}} \sigma$, where $n_{\mathrm{g}}$ is the neutral gas density (related to the pressure from the ideal gas law) and $\sigma$ is the ion-neutral charge exchange cross-section. At a pressure of $0.04 \mathrm{~Pa}$, this gives a mean free path of about $17 \mathrm{~cm}$, while at $0.3 \mathrm{~Pa}$, this becomes $2.3 \mathrm{~cm}$. Thus as the pressure increases the fraction of accelerated ions in the downstream region is significantly reduced.

Figure $9(b)$ shows the spatial dependence of the IEDF in the downstream region for a pressure of $0.04 \mathrm{~Pa}$. Here the higher energy ion population is observed at each axial location, becoming more distinct further downstream. At $z=0$, a definite peak exists at just under $40 \mathrm{~V}$, consistent with previous measurements of the plasma potential [20]. For $z<0 \mathrm{~cm}$ previous measurements show the plasma potential within the downstream region begins to decay [20]. As the plasma potential drops, a lower energy ion population becomes present in the IEDF, increasing in magnitude until at $z=7.5 \mathrm{~cm}$ the IEDF now shows a peak centred on the local plasma potential at this point. For $z>15 \mathrm{~cm}$ a significant higher energy ion population is clearly seen, with a range of energies between about $25-45 \mathrm{~V}$. Previous ion beam studies within the same reactor [8] (but operating under different conditions) have shown IEDFs with very clearly defined peaks, whereas here we observe higher energy populations with a much broader range of energies [17]. This is presumably related to a combination of the plasma potential profile present and the operating pressure, and will be explored further in section 4.5.

Radial measurements of the IEDF and current collected are shown in figure 10 for pressures of $0.04 \mathrm{~Pa}$, and $0.36 \mathrm{~Pa}$, respectively. These measurements are taken with RFEA (A) inserted into the side port of the diffusion chamber, and with its orifice facing the source region. Figure 10(a) shows that a higher energy ion population is present at all radial positions for the low pressure case, while the high pressure case in figure $10(c)$ shows no evidence of an ion beam. Figures $10(b)$ and $(d)$ show the total collected RFEA current for both pressures cases. While a slight asymmetry exists for the righthand side of the low pressure case, both radial current profiles are approximately symmetric. The total collected current for the low pressure case is about 3 times larger than that for the higher pressure case. Also shown in figure $10(b)$ is the ion beam current (open triangles, which have been scaled by a factor of 2 for clarity) at each radial position. The definition of the ion beam current follows that previously used [40]. The IEDF is decomposed into a sum of two Gaussians, one centred on the local plasma potential, and a second higher energy peak. The RFEA current that is measured at the discriminator voltage equal to the location of this second peak is then defined as the ion beam current. This approach serves as an estimate only, for two main reasons: (1) the IEDFs show broad distributions with no clearly defined second peak, so that interpretation of the ion beam is complicated, (2) if the beam diverges, the RFEA cannot be easily rotated to always remain normal to the incoming flux of ions.

The ion beam current in figure $10(b)$ shows a double humped shape with peaks at around $\pm 5 \mathrm{~cm}$, as well as showing
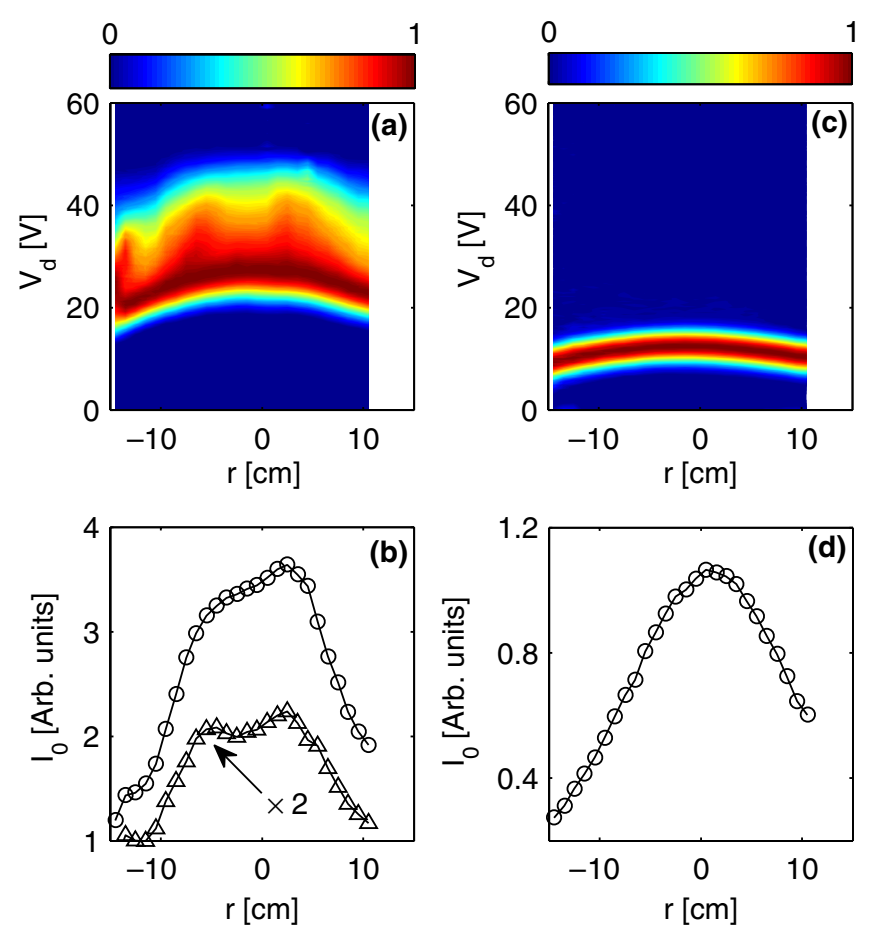

Figure 10. (a) IEDFs as a function of radial position (at $0.04 \mathrm{~Pa}$ ), taken downstream (at $z=15 \mathrm{~cm}$ ) with RFEA (A) inserted into a side port in the diffusion chamber and with its orifice facing the source region. (b) Total ion current (open circles) and beam current (open triangles) for the cases in $(a)$. The ion beam current has been scaled by a factor of 2 for clarity. (c) IEDFs as a function of radial position (at $0.36 \mathrm{~Pa}$ ). ( $d$ ) Total ion current (open circles) for the cases in $(c)$. The maximum magnetic field for cases $(a)-(d)$ (with just the soruce coil on) is $2.1 \mathrm{mT}$.

that most of the beam is contained within the region between -10 and $10 \mathrm{~cm}$ (similar to that for the total current). This allows an estimate of the beam divergence to be made. The average divergence, $\theta$, can be found from $\theta=\arctan \left[\left(R_{2}-\right.\right.$ $R_{\mathrm{S}}$ )/L], where $R_{\mathrm{S}}$ is the radius of the source tube and $R_{2}$ is the radius of the beam at a distance $L(15 \mathrm{~cm}$ from source tube exit to probe location) downstream from the source exit. If we assume $R_{2} \approx 10 \mathrm{~cm}$, then we get an average divergence of about $\theta \approx 12^{\circ}$. This is similar to divergence estimates made for ion beams formed in double-layer plasmas [5]. Observation of the last field line that leaves the source tube (thick red line online in figure 1) shows that this field line ends at the diffusion chamber wall (radius of about $16 \mathrm{~cm}$ ) after a distance of $15 \mathrm{~cm}$. Thus this field line has an average divergence of about $31^{\circ}$. This suggests that the ion beam is not following the field lines and that it might have detached from the magnetic field, although a more detailed 2D study would be needed to confirm this.

\subsection{Formation of multiple ion beam regimes}

In figure 4( $a$ ) a contour plot of the IEDFs for different magnetic fields was presented, where an ion beam was identified as occurring simultaneously with the low-field density peak in figure 3. This contour plot was shown for magnetic fields up to $3.7 \mathrm{mT}$. From figure 3 , as the magnetic field is further increased from this point, the density begins to rise again 


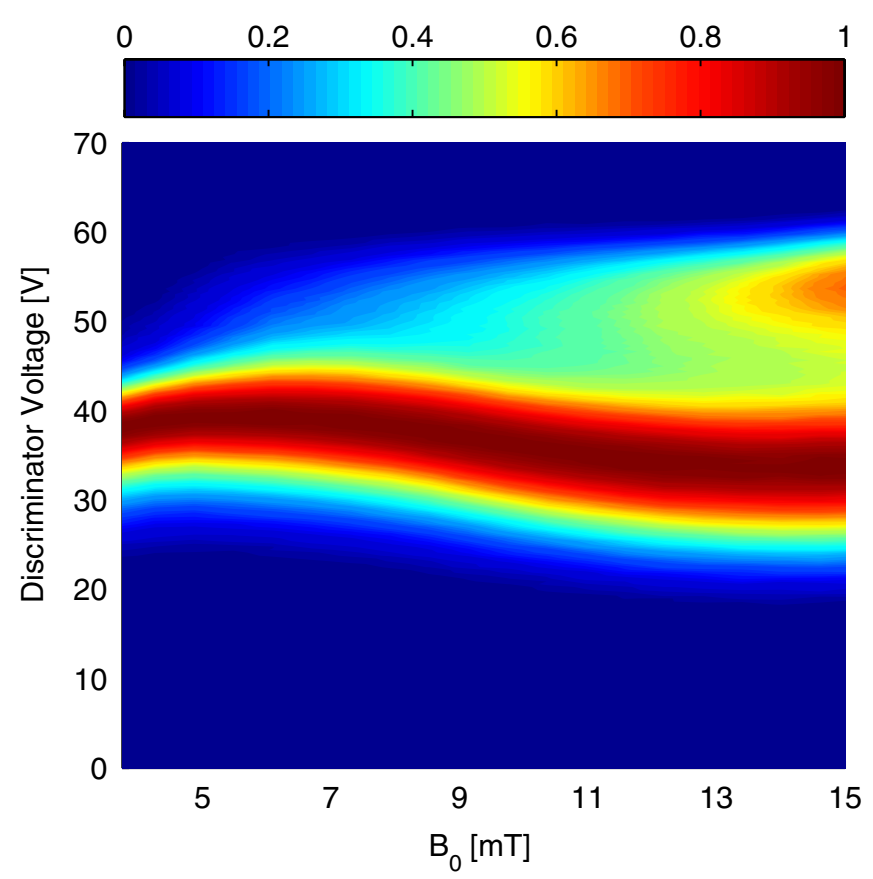

Figure 11. IEDFs as a function of the maximum applied magnetic field for the cases in figure 3 with $B_{0}>3.7 \mathrm{mT}$, taken downstream (at $z=10 \mathrm{~cm}$ ) with the 'dogleg' RFEA for a pressure of $0.04 \mathrm{~Pa}$.

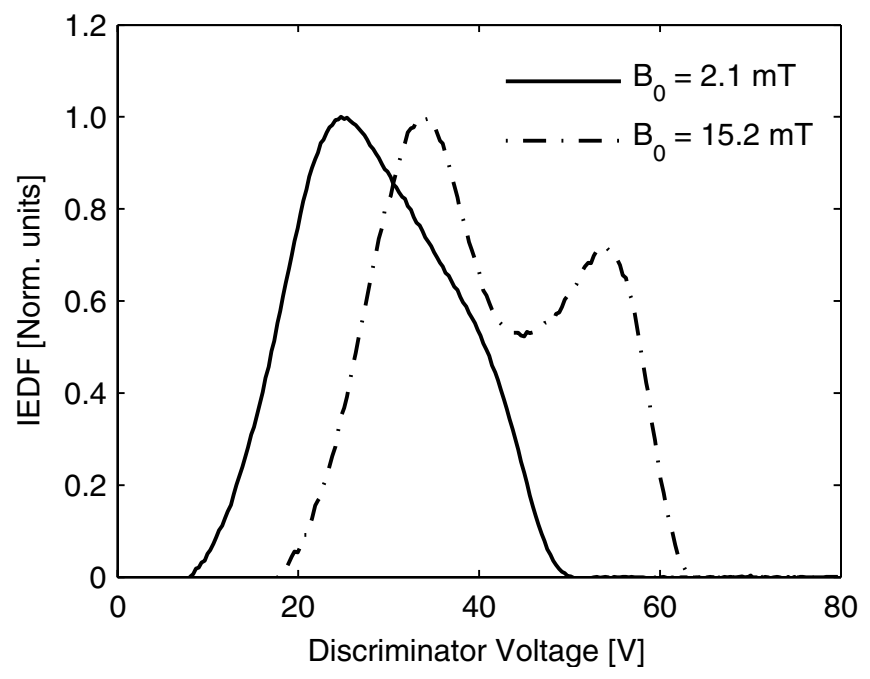

Figure 12. IEDFs at $B_{0}=2.1 \mathrm{mT}$ (solid line) and $B_{0}=15 \mathrm{mT}$ (dashed line) taken downstream (at $z=10 \mathrm{~cm}$ ) with the 'dogleg' RFEA for a pressure of $0.04 \mathrm{~Pa}$.

(although not as sharply as the low-field case). Figure 11 shows the IEDFs for these cases (that is for magnetic fields between $3.7 \mathrm{mT}<B_{0}<15 \mathrm{mT}$ ). At a magnetic field of around $5 \mathrm{mT} \mathrm{a}$ higher energy ion population is seen with energies of around $50-55 \mathrm{~V}$. This higher energy population becomes clearer and more distinct as the magnetic field is further increased, until for fields of around 13-15 $\mathrm{mT}$ a distinct second peak is observed in the IEDFs. At $15 \mathrm{mT}$ this second peak is centred at $55 \mathrm{~V}$, some $20 \mathrm{~V}$ higher than the local plasma potential of $35 \mathrm{~V}$. The IEDF at this magnetic field is plotted in figure 12, together with the IEDF occurring at the peak of the low field mode $(2.1 \mathrm{mT})$. As is seen, the high field IEDF shows two clearly distinct peaks, while the low field case is more distributed, skewed towards higher energies. Both the plasma and beam potentials are some 15-20 V larger for the higher field case. It is also interesting to note that from figure 11 this second ion beam regime begins for magnetic fields of around $5 \mathrm{mT}$. From figure 3 this is also close to the region where the density begins rising for the second time. In a previous publication [19] a similar transition was noted at $5 \mathrm{mT}$, where it was suggested that as the magnetic field was increased, the ion gyroradius would decrease until at around $5 \mathrm{mT}$ it was equal to the source tube radius. Further increases in magnetic field would reduce the gyroradius further, resulting in a decreased radial plasma loss rate. Since the source tube radius here is the same as that in [19], then if we assume a similar ion temperature, at $5 \mathrm{mT}$ the gyroradius would indeed be close to the source tube radius. This suggests this second ion beam regime is related to a similar phenomenon as that reported before.

Previous work [33] has shown that during the low-field density peak in figure 3 (and hence in the first ion beam regime) helicon wave behaviour has been measured within the source region. The second ion beam regime for the higher magnetic field produces upstream plasma densities and potentials (as well as ion beam behaviour and magnetic field strengths) similar to other studies, and it is interesting to ask whether this case is also a helicon mode. Although no B-dot probe measurements were made for this case, they were made for another case which gives similar densities and magnetic fields (as well as similar IEDF behaviour). Consistently, no wave signatures were observed in either the upstream or downstream regions. In the simplest case, from the dispersion relation for a plane unbounded helicon wave [22], the wavelength, $\lambda_{z}$, is given by

$$
\lambda_{z}=\sqrt{\frac{2 \pi B_{0}}{q \mu_{0} f n_{0}}},
$$

where $q$ is the electron charge magnitude, $f$ is the wave frequency, $\mu_{0}$ is the permeability of free space, $B_{0}$ is the magnetic field strength and $n_{0}$ is the plasma density. Using densities and magnetic fields from figure 3, for the low-field case $\left(B_{0}=2.1 \mathrm{mT}\right)$, equation (1) gives an axial wavelength of $18.6 \mathrm{~cm}$, which is approximately twice the length of the antenna (and is a well-known matching conditions for efficient antenna/plasma coupling [41]). For the high field case $\left(B_{0}=\right.$ $15 \mathrm{mT}$ ), this gives a wavelength of about $72.9 \mathrm{~cm}$, which we note is larger than the entire length of the experimental reactor. Since this wavelength cannot be accommodated in the reactor, it would thus seem as if the high field case $(>5 \mathrm{mT})$ is not in a helicon mode, as the density is too small to sustain the wave mode. Therefore this second ion beam regime is most likely in an inductive mode. A more exact estimate has been previously made [33] (at a slightly higher pressure of $0.08 \mathrm{~Pa}$ ) using the dispersion relation for cylindrically bounded helicon waves, and a similar conclusion is obtained.

As mentioned above, the second ion beam regime has similar densities, potentials and IEDF behaviour to that of other studies involving double layers [2-4]. While the reactors in these studies are somewhat similar to the present reactor, the densities are slightly lower than those occurring during 


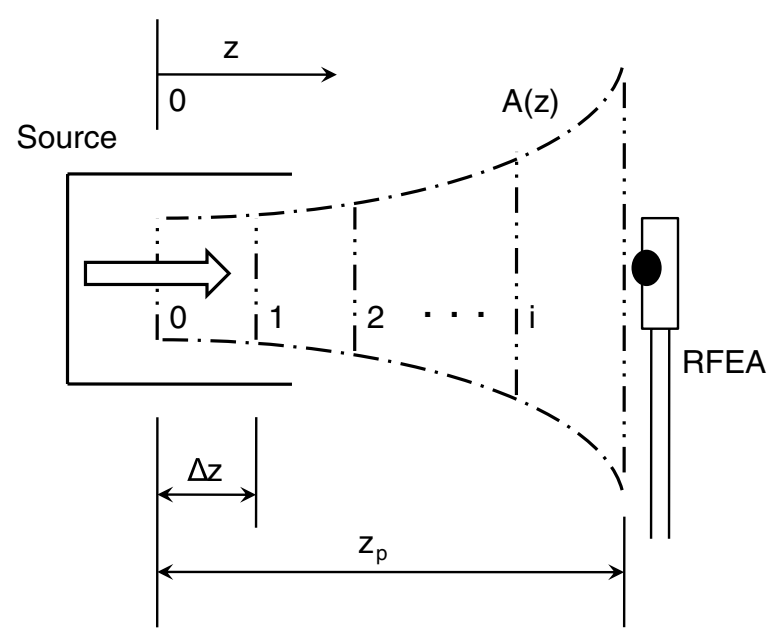

Figure 13. Schematic illustrating the model used. Ions in the source region are produced at point 0 and travel downstream. An ideal probe (which does not perturb the plasma) is located at $z_{\mathrm{p}}$, and collects the flux of ions that have flowed downstream.

the low-field mode here, and are thought to be inductively coupled [4], not helicon wave coupled. By contrast, in the first ion beam regime, helicon wave behaviour has been measured [33], so the plasma seems to be wave coupled. Based on the measurements of the IEDFs in figure 12, and the characterization in section 4.3 , the first ion beam regime, while showing some similarities, is clearly different from both the second ion beam regime and from previous double-layer studies, and seems uniquely related to the appearance of the low-field helicon mode (as seen in figure 4 and section 4.1).

\subsection{Modelling of the IEDFs}

Observation of figure 12 shows that the low-field and high-field IEDFs have very different shapes, and in particular the highfield case shows a definite second higher energy peak. The plasma potential profile has previously been measured [8] for conditions giving very similar ion beam behaviour to the highfield case. Here the plasma potential dropped by about $20 \mathrm{~V}$ over a distance of less than $10 \mathrm{~cm}$, before becoming roughly constant within the diffusion chamber. For the low-field case the potential changes much more gradually [20], with a drop of $20 \mathrm{~V}$ over about $20 \mathrm{~cm}$. We might wonder then if the shape of the IEDF is determined by the gradient of this drop, especially if the length scale for this change is similar to or larger than the mean free ion-neutral collision length.

To illustrate the above argument we make use of a simple analytical model, a schematic of which is shown in figure 13 (a model somewhat similar to the one presented here has been used in [17]). Ions are generated in the upstream region at $z=0$ and travel towards the downstream chamber, where due to divergence or collisions, the cross-sectional area of the flow can change. It is assumed that no ionization occurs in the downstream region, and that no loss processes occur (that is the plasma is confined to the area profile $A(z)$ ). It is also assumed that no ion flux travels upstream. An ion born in the upstream region has a potential energy of $V_{\mathrm{p}}(0)$, since if a grounded RFEA were inserted here, the ion would fall through the full potential to ground. We assume the probe to be ideal in the sense that it does not perturb the plasma in any way. Ions which enter from the source region $(z=0)$, travel downstream with the initial potential energy they were born with. However, ionneutral collisions along the propagation path (mainly charge exchange collisions as discussion in section 4.3) destroy this initial potential energy, and the ion gains a new potential energy associated with the plasma potential at the location it suffered a collision. Within the model, the plasma potential profile is set as a 'parameter' (see below).

Because of a number of factors, the IEDFs measured with the RFEA have a significant energy spread. This spread is a result of a combination of factors: (1) broadening of the IEDF due to rf modulation of the sheath in front of the RFEA, (2) finite temperature distribution of the ions, (3) ion-neutral collisions which scatter the ions, (4) divergence of the ion beam, (5) Energy resolution limits of the RFEA, (6) acceptance angle effects of the RFEA (the RFEA collects all ions within a certain solid angle). To avoid the need for a complicated model to describe all of these features, a simplified approach is used. It is assumed that the flux of ions carries with it a certain energy spread, chosen to be a Gaussian centred on the local plasma potential. Any ions that suffer collisions keep the same energy spread, but the Gaussian now becomes centred on the plasma potential at the location of the collision.

The energy spread function, $\bar{f}$, is chosen so that

$$
\int_{0}^{\infty} \bar{f}\left[V_{\mathrm{p}}(z)-V\right] \mathrm{d} V=1,
$$

where $\bar{f}$ is a normalized function as given above, $V_{\mathrm{p}}(z)$ is the plasma potential at the axial location $z$ and $V$ is an integration variable representing potential energy. From figure 13 , position $z=0$ is defined as the point where ions are produced and then move downstream. The total flux, $\bar{\Gamma}_{0}$ (which we have normalized to an amplitude of 1), of ions at this point can then be written as

$$
\bar{\Gamma}_{0}=\int_{0}^{\infty} \bar{f}\left[V_{\mathrm{p}}(0)-V\right] \mathrm{d} V=\beta_{0},
$$

where $\beta_{0}=\int_{0}^{\infty} \bar{f}\left(V_{p_{0}}-V\right) \mathrm{d} V$, and $V_{\mathrm{p}_{0}}$ is the plasma potential at position 0 . Due to ion-neutral collisions, which have a mean free path $\lambda$, a fraction $\mathrm{e}^{-\Delta z / \lambda}$ of this flux makes it to position 1 in figure 13 without suffering a collision, while a fraction $\left(1-\mathrm{e}^{-\Delta z / \lambda}\right)$ does suffer a collision. Due to the collisions this fraction now has an energy distribution centred on the plasma potential at position 1 . Because of the assumptions made in the model, the total flux at all axial locations must be conserved, but due to collisions, portions of this total flux now have a different potential energy. Thus the total flux at 1 is

$$
\bar{\Gamma}_{1}=\beta_{0} \mathrm{e}^{-\Delta z / \lambda}+\beta_{1}\left(1-\mathrm{e}^{-\Delta z / \lambda}\right),
$$

where $\beta_{1}=\int_{0}^{\infty} \bar{f}\left(V_{\mathrm{p}_{1}}-V\right) \mathrm{d} V$, and $V_{\mathrm{p}_{1}}$ is the plasma potential at position 1. If we proceed in this way (noting that the total flux must be conserved at each location), then we can write the flux at point $i$ as

$$
\bar{\Gamma}_{i}=\beta_{0} \mathrm{e}^{-\mathrm{i} \Delta z / \lambda}+\left(1-\mathrm{e}^{-\Delta z / \lambda}\right) \sum_{j=1}^{i} \beta_{j} \mathrm{e}^{-(i-j) \Delta z / \lambda},
$$


where $\beta_{j}=\int_{0}^{\infty} \bar{f}\left(V_{\mathrm{p}_{j}}-V\right) \mathrm{d} V$, and $V_{\mathrm{p}_{j}}$ is the plasma potential at position $j$. To write this equation in integral form, we note that $1-\mathrm{e}^{-\Delta z / \lambda} \approx \Delta z / \lambda$, which in the limit $\Delta z \rightarrow \mathrm{d} z$ becomes exact. Thus, by making use of definitions of the $\beta$ 's given above, we obtain

$$
\begin{aligned}
& \bar{\Gamma}\left(z_{\mathrm{p}}\right)=\mathrm{e}^{-z_{\mathrm{p}} / \lambda}\left\{\int_{0}^{\infty} \bar{f}\left[V_{\mathrm{p}}(0)-V\right] \mathrm{d} V\right. \\
& \left.\quad+\int_{0}^{z_{\mathrm{p}}}\left(\int_{0}^{\infty} \bar{f}\left[V_{\mathrm{p}}(z)-V\right] \mathrm{d} V\right) \mathrm{e}^{z / \lambda} \frac{\mathrm{d} z}{\lambda}\right\} .
\end{aligned}
$$

This represents the total flux at location $z_{\mathrm{p}}$. Note that since the total flux is conserved, and since we initially assumed a flux magnitude of 1 , the solution of equation (6) at any location $z_{\mathrm{p}}$ is equal to 1 . To find the contribution to the total flux from flux elements with potential energies between 0 and some specified voltage $V_{\mathrm{d}}$, we replace the limit $\infty$ by $V_{\mathrm{d}}$, and thus obtain

$$
\begin{gathered}
\bar{\Gamma}\left(z_{\mathrm{p}}, V_{\mathrm{d}}\right)=\mathrm{e}^{-z_{\mathrm{p}} / \lambda}\left\{\int_{0}^{V_{\mathrm{d}}} \bar{f}\left[V_{\mathrm{p}}(0)-V\right] \mathrm{d} V\right. \\
\left.+\int_{0}^{z_{\mathrm{p}}}\left(\int_{0}^{V_{\mathrm{d}}} \bar{f}\left[V_{\mathrm{p}}(z)-V\right] \mathrm{d} V\right) \mathrm{e}^{z / \lambda} \frac{\mathrm{d} z}{\lambda}\right\} .
\end{gathered}
$$

Now an RFEA typically works as follows: if the discriminator voltage is set to $V_{\mathrm{d}}$, then all ion flux with a potential energy greater than this will enter the probe. Thus the flux that the RFEA sees, $\bar{\Gamma}_{T}$, can be written as

$$
\bar{\Gamma}_{T}\left(z_{\mathrm{p}}, V_{\mathrm{d}}\right)=1-\bar{\Gamma}\left(z_{\mathrm{p}}, V_{\mathrm{d}}\right) .
$$

Note that if $V_{\mathrm{d}} \rightarrow \infty$ then no ions should enter the probe, while if $V_{\mathrm{d}}=0$, all ions should enter the probe. A check of equation (8) with these limits shows this is indeed true. The IEDF is then given by the derivative of equation (8) with respect to $V_{\mathrm{d}}$. Although the RFEA can measure the local value of the flux, we cannot account for radial variations in this model. Thus the flux per unit area is assumed constant, and since each IEDF will be normalized, this area need not be known. As mentioned above, a Gaussian form is used for the normalized energy distribution, given by

$$
\bar{f}\left[V_{\mathrm{p}}(z)-V\right]=\sqrt{\frac{\alpha}{\pi}} \mathrm{e}^{-\alpha\left[V_{\mathrm{p}}(z)-V\right]^{2}},
$$

where $\alpha$ is defined as the energy-width parameter (since it controls the width of the Gaussian). Note that there is a slight subtlety regarding the use of a Gaussian form for the energy distribution, since mathematically it is possible to obtain negative energies. If, however, the local plasma potential, $V_{\mathrm{p}}(z)$, is sufficiently greater than 0 , and if the energy spread parameter, $\alpha$, is sufficiently large, then the contribution from these negative energies is negligible, and so equation (2) can still be approximately satisfied. To investigate the shape of the IEDFs, we make use of two test cases for the plasma potential profile. We use a profile described by the following function:

$$
V_{\mathrm{p}}=V_{\mathrm{p}_{0}}\{a+b \tanh [c(z+d)]\},
$$

where $V_{\mathrm{p}_{0}}$ is the amplitude and $a, b, c$ and $d$ are coefficients. This equation is plotted in figure 14(a) for two test cases, a sharply varying profile $c=100$ and a gradual varying profile
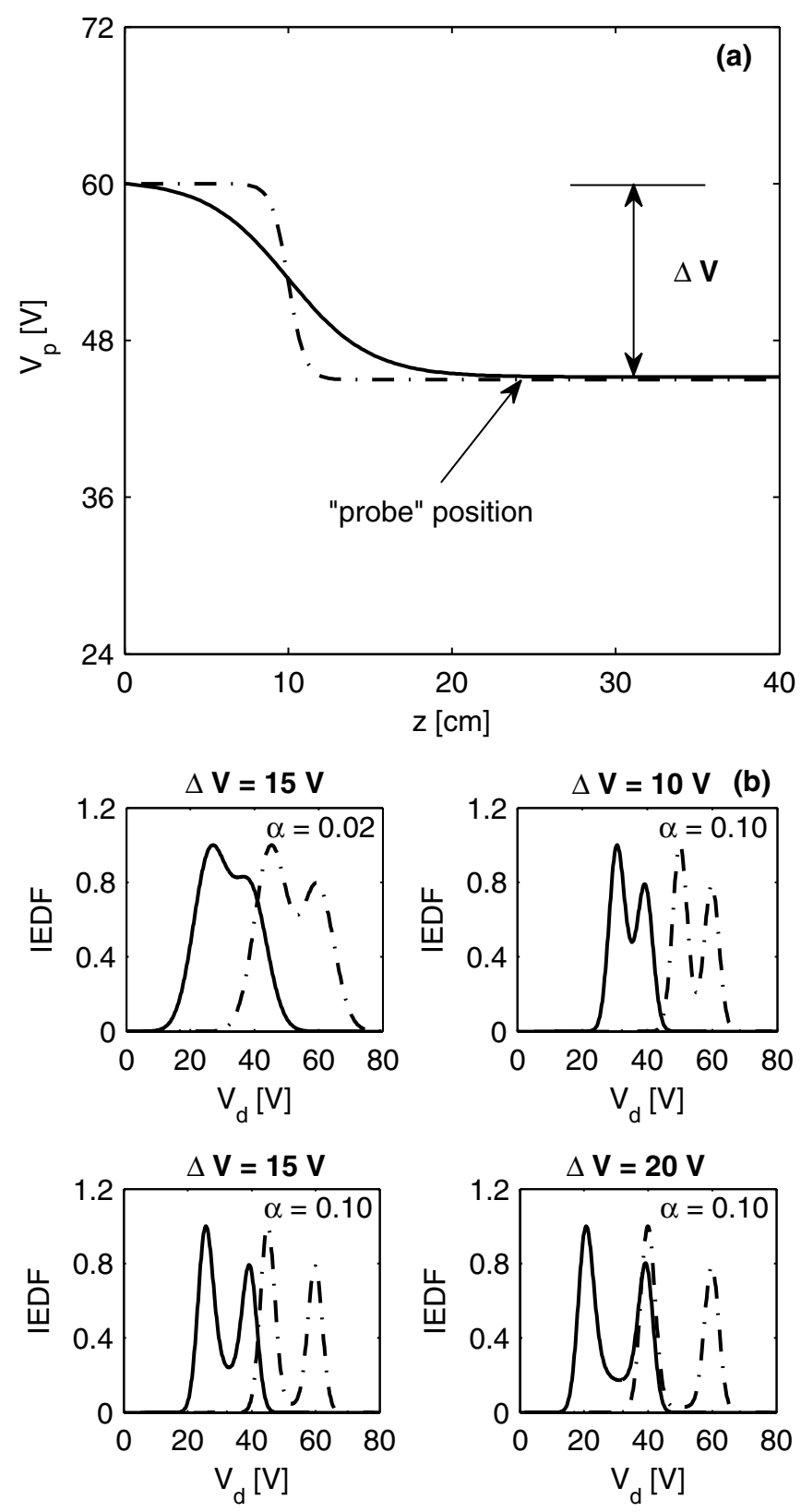

Figure 14. (a) Normalized plasma potential profiles used for the model, with $c=20$ (solid line) and $c=100$ (dashed line) for the fitting parameter in equation (10). Also shown is the probe location, and the definition of the total potential drop $\Delta V$. (b) 'Model-experiments' performed with varying values of the energy-width parameter, $\alpha$, and potential drop $\Delta V$, for the plasma potential profiles in $(a)$. The pressure is $0.04 \mathrm{~Pa}$ for all tests, and the potential amplitude in equation (10) is $V_{\mathrm{p}_{0}}=60 \mathrm{~V}$. The IEDFs for the case with $c=20$ have been shifted down by a value of $20 \mathrm{~V}$ to aid clarity. For all cases $d=0.1$, while for $\Delta V=10 \mathrm{~V} a=0.917$ and $b=0.083$, for $\Delta V=15 \mathrm{~V} a=0.875$ and $b=0.125$ and for $\Delta V=20 \mathrm{~V} a=0.8325$ and $b=0.1675$.

$c=20$ (the other coefficients are the same for both cases). The case with $c=100$ produces a sharp drop, $\Delta V$, in the plasma potential over a very narrow distance $(1 \mathrm{~cm})$, while the case with $c=20$ gives the same drop over a much larger distance $(10 \mathrm{~cm})$. A probe is inserted at $z=24 \mathrm{~cm}$ facing the upstream region. At this location the same potential drop has occurred for both test cases. A number of 'model-experiments' are 
then performed, as shown in figure 14(b). In the first 'modelexperiment' (top left figure), the energy-width parameter is set to $\alpha=0.02$, which gives a width similar to that seen in the real experiment. Other 'model-experiments' are conducted by varying the potential drop $\Delta V$, and the energy-width parameter. In the figures the IEDFs for the case with $c=20$ have been shifted down by $20 \mathrm{~V}$ so as to aid clarity. In all cases, the IEDFs for the case with $c=20$ (bold solid line) show a much larger energy spread, especially for the 'modelexperiments' with $\alpha=0.02$ (top left figure), where the IEDF produced is similar to that for the low-field case in figure 5. The case with $c=100$ has a more defined IEDF (bold dashed line) with two clear peaks, similar to that for the high field case in figure 12. Increasing $\alpha$ to 0.1 (hence decreasing the width of the energy distribution Gaussian) makes the peaks more distinct for $c=20$, but there is still an energy spread present between the peaks that is absent from the $c=100$ case. This is true for all other 'model-experiments'.

To investigate the effect of the collision mean free path we perform two further 'model-experiments', where we use the sharp potential profile $(c=100)$ and an energy-width $\alpha=0.02$. In the first 'model-experiment' we investigate the effect of the neutral gas pressure on the IEDFs. This is shown in figure $15(a)$. At low pressures $(<0.05 \mathrm{~Pa})$, there is almost no ion beam decay so that the RFEA sees only a single high energy peak centred on the beam potential, and only a very small lower energy peak (out of the field of view in figure 15(a)). As the pressure is increased (and hence as the mean free path is decreased) this beam begins to rapidly decay, until the lower energy population becomes dominant and only a small high energy tail remains. For pressures around 0.2-0.3 Pa, even this tail is almost gone, so that for higher pressures no ion beam exists. Note, however, that the same potential drop still exists, but there is no measurable ion beam. This is because charge exchange collisions have attenuated the beam to such an extent that it is no longer discernable in the downstream region. Thus even though a sharp potential gradient might exist, no ion beam might be detected since the length scale on which it decays is too short. In real experiments the potential drop is seen to decrease with pressure $[12,38]$ (and the energy-width often changes as well), but the above argument still applies.

In the second 'model-experiment', the pressure is fixed at $0.04 \mathrm{~Pa}$ (with the same energy-width of $\alpha=0.02$ ) and the IEDFs are investigated for varying probe positions. For positions above the sharp drop $(z<10 \mathrm{~cm})$, the IEDF shows a single peak at the local plasma potential. As the probe moves closer to the drop a small low energy population develops (out of the field of view in figure 15(b)), until for $z>20 \mathrm{~cm}$ the ion beam begins to rapidly decay, and the low energy population dominates. This qualitative behaviour matches well with the experimental results from other studies [3,5]. In the experimental measurements in figure $9(b)$ the higher energy part of the IEDF does not decay as quickly as in the model since the plasma potential profile is not constant in the diffusion chamber, so that an ion suffering a collision can be re-accelerated from this location to become a higher energy ion downstream of this location (although not with the full potential drop from the source region).
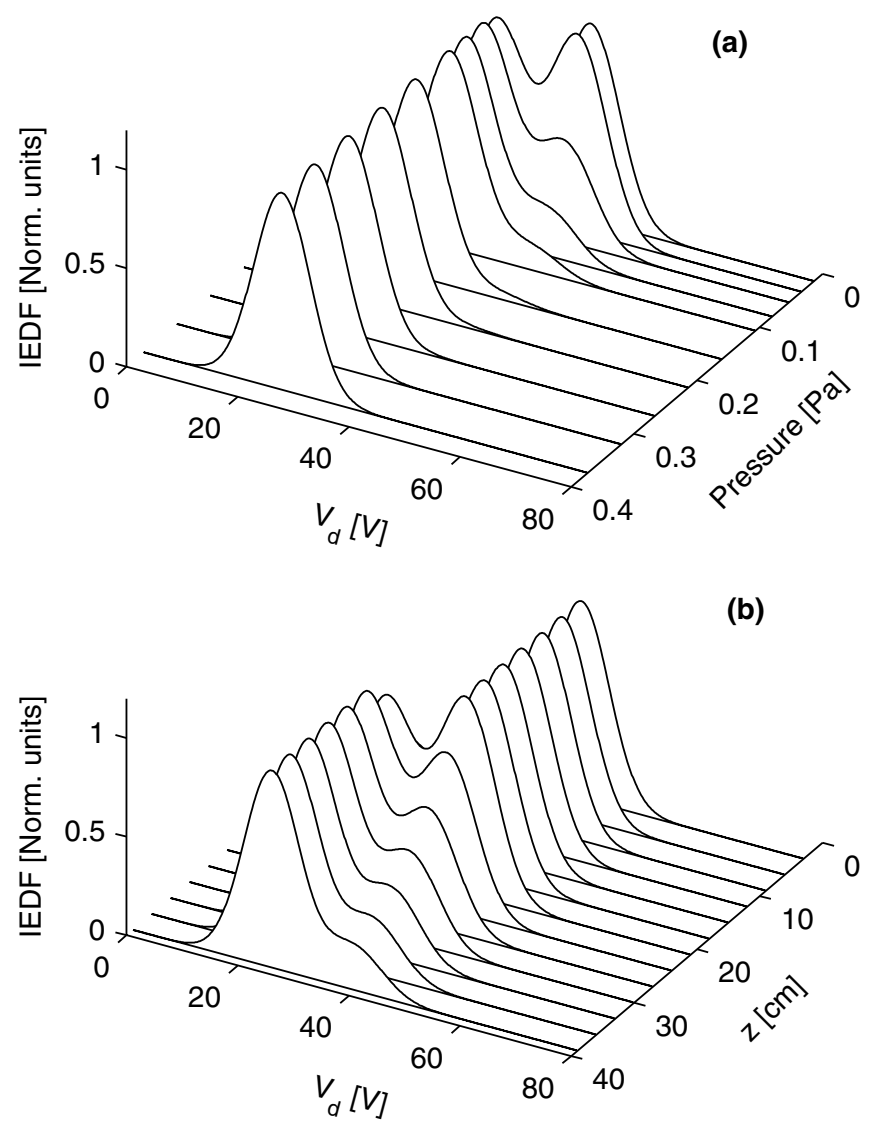

Figure 15. (a) IEDFs from the model as a function of neutral gas pressure at $z=24 \mathrm{~cm}$ in figure 14(a). (b) IEDFs from the model as a function of axial position for a gas pressure of $0.04 \mathrm{~Pa}$. For both (a) and (b), $\alpha=0.02, d=0.1, \Delta V=15 \mathrm{~V}$ (so that $a=0.875$ and $b=0.125$ ), and the sharply varying plasma potential profile $(c=100)$ in figure $14(a)$ is used.

The above arguments illustrate the fact that the shape of the IEDF is controlled by the plasma potential profile, the energywidth parameter, and the neutral gas pressure. For a more gradually varying plasma potential profile, with a length scale similar to or larger than the ion-neutral mean free collision length, a larger energy spread is seen in the IEDFs when compared with profiles that vary more sharply. We also note in passing that the IEDFs from the model show good qualitative agreement with those seen in experiment $[3,4,6,8]$, suggesting that an extension of the present model could be made to more usefully complement experimental studies. In particular if a better model could be developed to predict the energy-width parameter, which at the moment needs to be set.

\section{Discussion}

The results presented in section 4 show that the ion beam exists simultaneously with the low-field helicon mode. This is most clearly seen in figure 4 , where a higher energy ion population appears in the IEDF at the same magnetic field at which the helicon mode is initiated, and then subsequently disappears at the same magnetic field at which the helicon mode vanishes. A similar result is obtained as the rf power is increased, where the ion beam appears at a critical power input, 
which corresponds to the same power needed to initiate the helicon mode. Additionally, for a range of different pressures, an ion beam is seen during the low-field modes (for pressures below $0.3 \mathrm{~Pa}$ ) as demonstrated in figure $9(a)$. These facts all suggest that the appearance of the low-field helicon mode in some way induces the formation of the ion beam.

In a previous publication [20], the plasma density profile during the low-field mode was measured and was found to strongly diverge, approximately following the magnetic field shape at $0.04 \mathrm{~Pa}$. A recent investigation [37] has found that at higher pressures $(0.36 \mathrm{~Pa})$ the plasma density even more closely follows the magnetic field profile, even if the field changes location (such as by using the exhaust coil in figure 1). Outside of the low-field mode however, the plasma density is more uniform, even though the magnetic field still diverges [20], suggesting that the plasma is not forced to follow the magnetic field. Since the density in these cases is low, the plasma skin depth in the system is quite large and plasma can be formed capacitively throughout the reactor. During the helicon mode however, the skin depth decreases and capacitive coupling is significantly reduced, and a large amount of power is deposited mainly within the source region, thus acting to preferentially increase the density in this region. As this plasma flows downstream it is confined by the magnetic field, and forced to undergo expansion as it diverges. As a result, this sets up a large density gradient between the upstream $(z<0 \mathrm{~cm})$ and downstream regions $(z>0 \mathrm{~cm})$. If for the sake of argument we assume Boltzmann electrons [24], then the plasma potential is related to the density by

$$
V_{\mathrm{p}}(z)=V_{\mathrm{p}_{0}}+T_{\mathrm{e}} \ln \frac{n(z)}{n_{0}}
$$

where $V_{\mathrm{p}_{0}}$ and $n_{0}$ are the plasma potential and density at $z=0$, respectively, and $T_{\mathrm{e}}$ is the electron temperature (in $\mathrm{eV}$ ). From equation (11), if the density is strongly varying, then a large potential change exists. Ions born in the upstream region will then travel downstream, accelerating along this potential profile, and if the pressure is sufficiently low, an ion beam will be present in the downstream region. This requirement of low pressure is necessary to ensure a sufficiently large mean free path length for ion-neutral collisions (so that the beam can be 'sustained' in the downstream region). At the ion energies typically seen in experiments $(0-20 \mathrm{eV})$ the upper limit for this condition is about $0.3-0.4 \mathrm{~Pa}$. For larger ion energies the collision cross-sections are smaller [39] so that this limit could be extended. Also, this limit applies to argon gas, so that other gases (which would have different cross-sections) could have a higher or lower limit [42].

We might wonder though if the low-field helicon mode is directly responsible for the ion beam creation, since this has not been ruled out in the discussion above. Previous work [33] has shown that by slightly changing the magnetic field strength and geometry at the source exit, a significant modification of the plasma density and plasma potential profiles can be obtained, in addition to changes to the IEDF. In particular, as the magnetic field in the downstream region is made more uniform, both the plasma density and potential start becoming approximately constant, and the ion beam disappears. However, the low-field mode is still present. Thus this implies that the helicon mode is not the direct source of the ion beam. Based on the discussion above then, the helicon modes role seems to be in preferentially creating a large density within the source region in the presence of a diverging magnetic field.

Although from the results in section 4 the ion beam occurs simultaneously with the low-field mode, what remains unclear is the lower and upper magnetic field limits for the low-field mode to be present. Since lower and upper limits exist for lowfield modes even in uniform magnetic fields where no ion beam forms, these limits seem related to the helicon mode formation itself, and not the ion beam. That is, as long as the low-field mode is present, an ion beam is present (although this statement need not be true in general). Measurements $[29,37]$ show that as the power or pressure increases, the density peaks become broader and higher. It is thought that the formation of these peaks is related to antenna coupling and plasma dispersion characteristics [37,43], but this is not yet well understood and requires further investigation.

We have shown that multiple ion beam regimes can exist with the same system geometry, pressure, and total power input, but with a larger magnetic field. The IEDFs for these regimes were shown to possess different shapes, which from the model developed is a function of the system pressure and gradient of the plasma potential profile. The common factor for both ion beam regimes is that the neutral gas pressure is low, and that a large density gradient exists between the upstream and downstream regions. The second ion beam regime (at $B_{0}=15 \mathrm{mT}$ ) was shown to have a density too low for the applied field for it to be helicon sustained, and so is most likely to be inductively coupled. If the exhaust coil is used together with the source coil (see figure 1), then for large enough currents no low-field peak is seen anymore, but a sharper transition is observed from a capacitive to an inductive mode (with densities similar to the high field ion beam regime). This particular magnetic field induced transition is then possibly due to the fact that the increased magnetic field reduces radial losses to the source tube walls which then allows the central density to increase, and hence the system can jump into an inductive mode (inductive discharges typically require a minimum density to be initiated [24]). This, however, is also an area of ongoing research.

\section{Conclusions}

In summary, we have performed a spatial and parametric characterization of the ion beam formed during a low-field helicon mode in the presence of a diverging magnetic field. The appearance of the ion beam and low-field mode are strongly correlated, implying that the beam is formed by the expanding plasma that is induced during the helicon mode. Within the mode the ion beam is present for a range of $\mathrm{rf}$ powers $(150-400 \mathrm{~W})$, and appears to show no power dependence (aside from a change in the measured current magnitude). The beam is only observed for pressures less than about $0.3 \mathrm{~Pa}$, after which the ion-neutral collision mean free path becomes too small to sustain a beam for any significant distances downstream. A second ion beam regime is identified for 
the same operating pressure and rf power, but with a larger magnetic field ( $>5 \mathrm{mT}$ ). This regime appears to operate in an inductive mode, with a lower plasma density but higher plasma potential compared with the low-field mode. Results from a simple analytical model show that the gradient of the plasma potential profile and neutral gas pressure are important factors in determining the general shape of the IEDF.

\section{References}

[1] Cohen S A, Siefert N S, Strange S, Boivin R F, Scime E E and Levinton F M 2003 Phys. Plasmas 102593

[2] Charles C and Boswell R W 2003 Appl. Phys. Lett. 821356

[3] Charles C and Boswell R W 2004 Phys. Plasmas 111706

[4] West M, Charles C and Boswell R W 2008 J. Propul. Power 24134

[5] Cox W, Charles C, Boswell R W and Hawkins R 2008 Appl. Phys. Lett. 93071505

[6] Takahashi K, Oguni K, Yamada H and Fujiwara T 2008 Phys. Plasmas 15084501

[7] Takahashi K, Igarashi Y and Fujiwara T 2010 Appl. Phys. Lett. 97041501

[8] Lafleur T, Charles C and Boswell R W 2009 Phys. Plasmas 16044510

[9] Virko V F, Virko Y V, Slobodyan V M and Shamrai K P 2010 Plasma Sources Sci. Technol. 19015004

[10] Chen F F 2006 Phys. Plasmas 13034502

[11] Fruchtman A 2006 Phys. Rev. Lett. 96065002

[12] Lieberman M A, Charles C and Boswell R W 2006 J. Phys. D: Appl. Phys. 393294

[13] Meige A, Boswell R W, Charles C and Turner M M 2005 Phys. Plasmas 12052317

[14] Kawamura E, Lichtenberg A J, Lieberman M A and Verboncoeur J P 2009 Phys. Plasmas 16122114

[15] Boswell R W, Marsch E and Charles C 2006 Astrophys. J. 640 L199

[16] Charles C, Boswell R W and Hawkins R 2009 Phys. Rev. Lett. 103095001

[17] Charles C, Boswell R W and Porteous R K 1992 J. Vac. Sci. Technol. A 10398

[18] Corr C S, Zanger J, Boswell R W and Charles C 2007 Appl. Phys. Lett. 91241501
[19] Charles C and Boswell R W 2007 Appl. Phys. Lett. 91201505

[20] Lafleur T, Charles C and Boswell R W 2010 Phys. Plasmas 17043505

[21] Takahashi K, Kaneko T and Hatakeyama R 2008 Phys. Plasmas $\mathbf{1 5} 072108$

[22] Boswell R W and Chen F F 1997 IEEE Trans. Plasma Sci. 251229

[23] Shoji T, Sakawa Y, Nakazawa S, Kadota K and Sato T 1993 Plasma Sources Sci. Technol. 25

[24] Lieberman M A and Lichtenberg A J 2005 Principles of Plasma Discharges and Materials Processing (Hoboken, NJ: Wiley)

[25] Berisford D F, Bengston R D and Raja L L 2010 Phys. Plasmas 17033503

[26] Goebel D M and Katz I 2008 Fundamentals of Electric Propulsion (Hoboken, NJ: Wiley)

[27] Chen F F 1992 J. Vac. Sci. Technol. A 101389

[28] Wang S J, Kwak J G, Kim C B and Kim S K 2003 Phys. Lett. A 313278

[29] Sato G, Oohara W and Hatakeyama R 2007 Plasma Sources Sci. Technol. 16734

[30] Degeling A W, Jung C O, Boswell R W and Ellingboe A R 1996 Phys. Plasmas 32788

[31] Chen F F 2008 IEEE Trans. Plasma Sci. 362095

[32] Chen F F and Torreblanca H 2009 Phys. Plasmas 16057102

[33] Lafleur T, Charles C and Boswell R W 2010 Phys. Plasmas 17073508

[34] Perry A, Conway G, Boswell R W and Persing H 2002 Phys. Plasmas 93171

[35] Kemp R E and Sellen J M 1966 Rev. Sci. Instrum. 37455

[36] Charles C, Degeling A W, Sheridan T E, Harris J H, Lieberman M A and Boswell R W 2000 Phys. Plasmas 75232

[37] Lafleur T, Charles C and Boswell R W 2011 Characterization of a helicon plasma source in low diverging magnetic fields J. Phys. D: Appl. Phys. 44055202

[38] Charles C 2005 Phys. Plasmas 12044508

[39] Phelps A V 1994 J. Appl. Phys. 76747

[40] Charles C, Boswell R W, Cox W, Laine R and MacLellan P 2008 Appl. Phys. Lett. 93201501

[41] Boswell R W 1984 Plasma Phys. Control. Fusion 261147

[42] Charles C, Boswell R W and Lieberman M A 2006 Appl. Phys. Lett. 89261503

[43] Cho S 2006 Phys. Plasmas 13033504 